\title{
Phase behaviour of colloids plus weakly adhesive polymers
}

\author{
R. Tuinier ${ }^{1,2, a}$, S. Ouhajji ${ }^{2,3}$, and P. Linse ${ }^{3}$ \\ 1 Laboratory of Physical Chemistry (SPC), Department of Chemical Engineering and Chemistry \& Institute for Complex \\ Molecular Systems (ICMS), Eindhoven University of Technology, Helix STO 2.49, P.O. Box 513, 5600 MB Eindhoven, The \\ Netherlands \\ 2 Van 't Hoff Laboratory for Physical and Colloid Chemistry, Department of Chemistry \& Debye Institute, Utrecht University, \\ Padualaan 8, 3584 CH Utrecht, The Netherlands \\ 3 Physical Chemistry, Department of Chemistry, Lund University, P.O. Box 124, S-22100, Lund, Sweden
}

Received 14 June 2016 and Received in final form 2 November 2016

Published online: 30 November 2016

(c) The Author(s) 2016. This article is published with open access at Springerlink.com

\begin{abstract}
The phase behaviour of a colloidal dispersion mediated by weakly adhesive polymers is considered. The polymers are depleted but are weakly adhesive and hence comprise a non-zero polymer concentration at the colloid's surface, in contrast to the classical assumption in depletion theories involving a zero polymer concentration at the surface. The theory is composed of a generalized free-volume theory for colloid-polymer mixtures and a self-consistent mean-field theory for polymers at surfaces. It is found that the weak adhesion of the polymers shifts the phase stability of the colloid-polymer mixtures to higher polymer concentrations as compared to assuming a full depletion effect. The predicted phase diagrams employing the new theory are consistent with experiments on mixtures of silica spheres coated with stearyl alcohol and polydimethylsiloxane in cyclohexane and with Monte Carlo simulation results.
\end{abstract}

\section{Introduction}

The interaction between colloidal particles and the phase stability of colloidal dispersions with added polymers depends strongly on the effective interactions between the polymer chains and the surface of the colloidal particles $[1-3]$. Typically, there is either negative adsorption (usually termed depletion) or positive adsorption (often denoted as adsorption). The definition of the adsorbed amount $\Gamma$ at a flat surface is $[2,4]$

$$
\Gamma=\int_{0}^{\infty}\left[\varphi(z)-\varphi_{b}\right] \mathrm{d} z,
$$

where $\varphi(z)$ is the polymer segment concentration as a function of the distance $z$ from the flat surface and $\varphi_{b}$ is the polymer segment bulk concentration. In case of polymer adsorption $(\Gamma>0)$, bridging flocculation is expected at low polymer concentration, while colloidal stability can be achieved when there is enough polymer to cover the surfaces of the particles $[1,2]$. The interaction between the particles and the stability of mixtures of adsorbing polymers and nanoparticles was considered for instance by Surve et al. [5].

In most depletion theories it is usually implicitly (and sometimes explicitly) assumed that $\varphi(z=0) \equiv 0[1,3,4]$.

\footnotetext{
a e-mail: r.tuinier@tue.nl
}

As long as $\Gamma<0$, negative adsorption and hence depletion effects are still expected, even when there is weak adhesion of the polymers to the surface. The case $\Gamma=0$ is a special condition that is difficult to realize experimentally. It corresponds to the case that the adhesive strength of the polymer segments to the surface exactly matches the loss of entropy that is associated with polymer adsorption. Positive adsorption $(\Gamma>0)$ takes place when the attraction is stronger than the entropy loss. In fig. 1 the various possibilities ranging from full depletion $\left(\varphi_{\text {surf }}=\varphi(z=0)=0\right)$, weak adhesion $\left(0<\varphi_{\text {surf }}<\varphi_{b}\right)$ to (strong) adsorption $\left(\varphi_{\text {surf }}(>)>\varphi_{b}\right)$ are sketched schematically. It is noted that the above holds for colloids mixed with polymer solutions, although the strength of adhesion also plays a key role for nanoparticle-polymer melt hybrids, see for instance Hooper and Schweizer [6]. The polymer density profiles $\varphi(z)$ corresponding to the various cases discussed are shown in fig. 2 .

Whenever $\Gamma<0$, the colloidal particles in mixtures of a colloidal dispersion with added polymer chains are surrounded by depletion zones with reduced polymer density $[7,8]$, originating from the entropy loss of polymers located close to the surfaces of the colloids [9]. When two such depletion zones overlap, colloidal particles attract each other $[10,11]$. As a result, the presence of nonadsorbing polymer chains leads to a depletion-mediated attraction between colloidal particles [3], which can be 


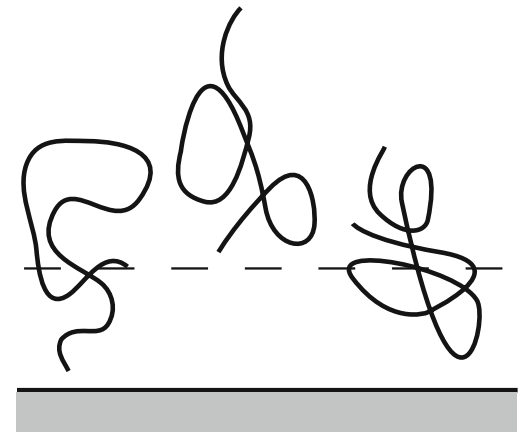

(a) full depletion

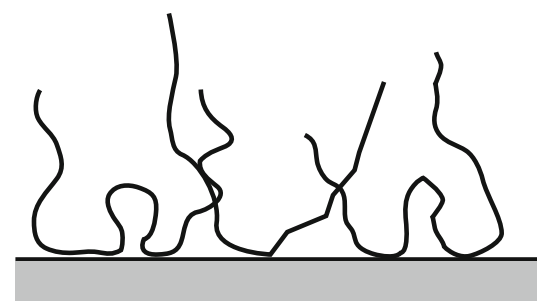

(c) weak adsorption

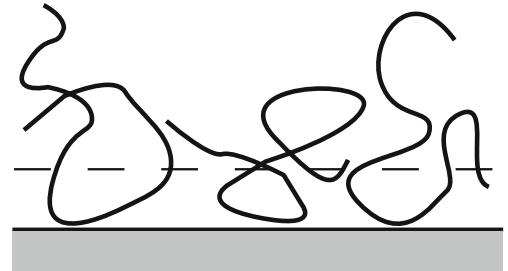

(b) weak adhesion

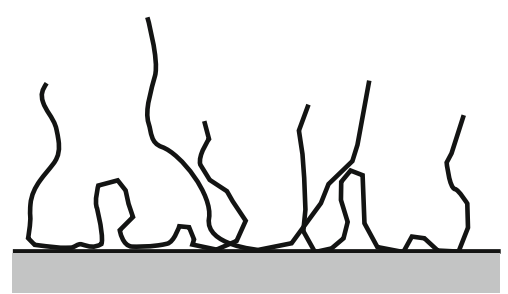

(d) full adsorption

Fig. 1. Polymers in solution near an interface for various adhesion strengths: (a) the polymer chains are fully depleted corresponding to the common boundary condition $\varphi(z=0) \equiv 0$, (b) the polymer chains weakly adhere to the surface but are still depleted (there is negative adsorption of polymer), (c) the polymer chains weakly adsorb and (d) the polymer chains strongly adsorb and form the characteristic train-loop-tail configurations in the adsorbed state. Note that the thickness of the depletion zone is indicated via the dashes in (a) and (b).

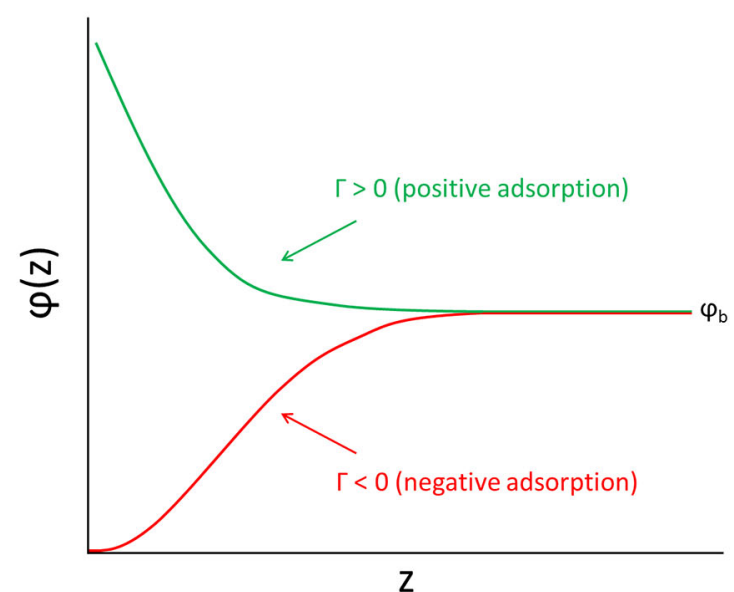

(a)

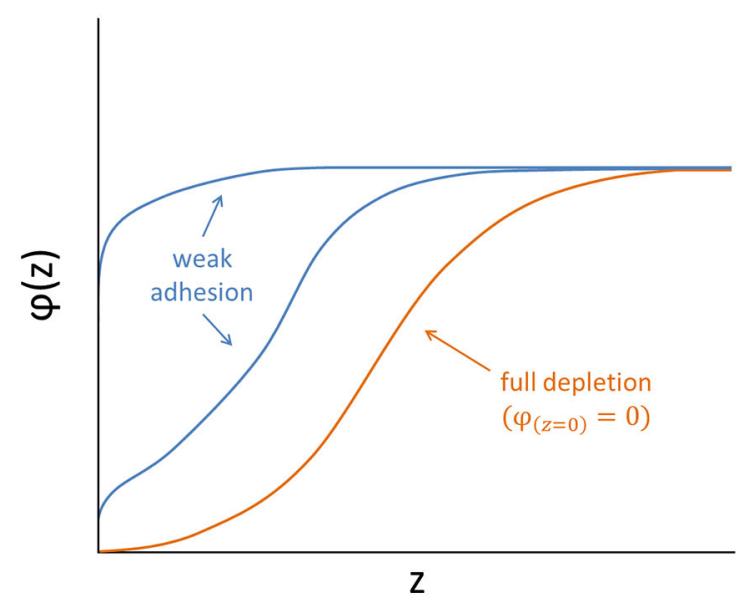

(b)

Fig. 2. Polymer density profiles $\varphi(z)$ as a function of the distance from the surface $z$ : (a) positive and negative adsorption and (b) weak adhesion and full depletion of polymers in solution near an interface.

measured using, for instance, atomic force microscopy, optical tweezers and total internal reflection microscopy [12]. Depletion forces can be used to tune the phase behaviour of colloidal dispersions [8], and depletion effects also have a pronounced influence on colloid dynamics [13]. This is relevant for the stability of colloid-polymer mixtures in industrial applications such as food [14] and high-performance photo-voltaic materials [15] as well as for understanding transport phenomena in these systems.
Mixtures of colloidal hard spheres (HS) and nonadsorbing polymers are of special fundamental interest. These are considered to be the ultimate model system in which the range (by choosing the polymer size) and strength (by varying the polymer concentration) of the attraction can be tuned independently. Phase diagrams of such systems have been studied experimentally in great detail [16-22]. Coexisting phases include colloidal gasliquid, fluid-solid and a three-phase coexistence region [17, 
18,20,23-25]. Generally, such colloidal spheres are characterized by their HS radius $a$, the polymers by their radius of gyration $R_{g}$, and the mixture by the polymer-colloid size ratio $q=R_{g} / a[26,27]$. The mixture of colloidal polymethylmethacrylate (PMMA) spheres and flexible polystyrene (PS) chains dispersed in cis-decalin $[8,18]$ has received special attention, and has been used as a testbed for the validity of various depletion theories. When phase transitions occur well below the polymer overlap concentration, the phase behaviour of the well-defined reference PMMA + PS reference mixtures could be described almost quantitatively by the free-volume theory (FVT) $[8,26]$. FVT is in close agreement with Monte Carlo computer simulations [28-31]. Whenever the polymer concentrations get close to or beyond the polymer overlap concentration, FVT deviates from experiments. This is due to the fact that the polymer concentrations required to induce phase transitions are at concentrations where polymeric interactions should be accounted for. Various methods have been employed to describe polymer physics effects onto depletion interaction, liquid structure and phase stability including self-consistent mean-field theory $[4,32,33]$, PRISM integral equation theory [34-36] and a Gaussian core model for the polymers [37-39]. The theoretical description for the full phase behaviour can be improved by using a generalized free-volume theory (GFVT), in which excluded-volume interactions between the polymer segments are included $[31,40]$. The theoretical predictions are in agreement with recent Monte Carlo computer simulations [41]. In practice, experimental studies reported in the literature are often performed using particles that are disperse in size to such a degree that crystallization is suppressed [42]. Hence, in some studies only colloidal gas-liquid coexistence is observed [21,43,44], as has been predicted by Fasolo and Sollich [45].

In this paper it is shown how weak adhesion of the polymer at the surface of the colloidal particles sensitively affects depletion effects. It is demonstrated how incorporation of weak adhesion can improve the description of the phase behaviour of colloid-polymer mixtures. The gist of the new theory is a non-zero polymer concentration at the colloid's surface [34,46], in contrast to the classical depletion approach involving a zero polymer concentration. It is known that adhesive contributions can modify depletion potentials [47]. A higher polymer surface concentration affects the phase stability region even when there is still negative adsorption: the location of the binodals shifts to higher polymer concentration as compared to the classical case. It is shown that this is consistent with experimental phase diagrams of mixtures of silica spheres, sterically stabilized with stearyl alcohol, and polydimethylsiloxane (PDMS) polymers in cyclohexane $[21,43,46]$.

\section{Methods}

\subsection{Experiments}

We have experimentally measured the phase diagram of silica spheres coated with stearyl alcohol mixed with
PDMS polymers in cyclohexane. Sterically stabilized silica spheres, with an apparent hydrodynamic diameter of $74 \pm 14 \mathrm{~nm}$, were synthesized as described previously [48]. PDMS (Fluka) with a weight-averaged molar mass of $95 \mathrm{~kg} / \mathrm{mol}$ and a molar mass dispersity (in terms of $\left.M_{\mathrm{w}} / M_{\mathrm{n}}\right)$ of 1.9 in cyclohexane $(99 \%$, Sigma-Aldrich) was used. The (averaged) radius of gyration of PDMS is close to $14.4 \mathrm{~nm}$ [49]. The molar mass of a DMS is $74 \mathrm{~g} / \mathrm{mol}$ [50], giving $N \approx 1284$ repeating units in PDMS. Dilute PDMS solutions in cyclohexane at room temperature can be characterized as a polymer solution with the Flory-Huggins polymer(P)-solvent(S) interaction parameter $\chi_{\mathrm{ps}} \approx 0.4$ [51]. Excluded-volume interactions between the polymer segments play a role for $\chi_{\mathrm{ps}}<1 / 2$. For this system $q=0.38$, which in theory allows for colloidal gas, liquid and crystalline phases. However, only gas-liquid phase separation was observed, as can be explained by size dispersity of the colloidal silica spheres [45], which in this case is about $20 \%$.

\subsection{Theory}

The theory used in this work consists of free volume theory for the phase behaviour of colloidal dispersions of spheres with added weakly adhesive polymer chains and a selfconsistent mean-field lattice theory (SCMFT) describing polymers at surfaces.

\subsubsection{Self-consistent mean-field theory}

Over the last few decades, SCMFT has become an increasingly powerful technique for studying inhomogeneous polymer systems $[2,52-55]$. The mean-field free energy is a central quantity in SCMFT, which encompasses mixing entropy, Flory-Huggins mixing enthalpy, local meanfield interactions, and a compressibility constraint. After minimizing the free energy using a self-consistent iterative protocol, thermodynamic equilibrium properties of the system can be obtained. In particular, SCMFT enables computing the equilibrium polymer segment density $\varphi(z)$ as a function of the distance from the surface $z$.

The mean-field theory, and more specifically the Bragg-Williams approximation, allows to formulate a free energy for a system in terms of two profiles. These are the measurable volume fraction profile $\varphi(z)$ and the complementary segment potential profile $u(z)$ distributions. These potentials can be viewed as external potentials and are used to obtain Boltzmann weights $G(z)=\exp (-u(z))$. All energy quantities are made dimensionless by normalising them with the thermal energy $k_{B} T$. The segment potentials are a function of the volume fractions in a FloryHuggins fashion. The segment potential takes into account the interaction of a given segment with its surroundings. These interactions are set by Flory-Huggins interaction parameters $\chi_{x y}$, wherein both $x$ and $y$ are segment or solvent or surface types. A positive $\chi$ value implies that the $x y$ contacts are less favourable than the average of $x x$ and yy contacts. Hence a sufficiently large positive value for 
$\chi_{x y}$ implies segregation of $x$ and $y$ if these were a binary mixture. Low values or negative values imply that such a mixture is miscible.

The potentials depend on the volume fractions, but the volume fraction profiles themselves are also a function of the segment potentials. The SCMFT method is characterised by the relation

$$
\varphi[\{u\}] \leftrightarrow u[\{\varphi\}] .
$$

This equation implies that the volume fractions can be computed from the potentials and vice versa. When the sets of potentials and volume fractions are consistent the solution is self-consistent. An additional required constraint used here is to fix the system to be incompressible and impose

$$
\sum_{x} \varphi_{x}(z)=1
$$

for each value of $z$.

In some cases (for instance for some applications of ideal polymers) it is possible to solve the self-consistent field solution analytically. Usually, the equations need to be solved numerically. Here the SCMFT method of Scheutjens and Fleer $[2,52]$ is followed that makes use of a lattice of sites in order to discretise the equations. Polymer chains are described using the freely-joined chain model. The characteristic length of a lattice site is taken equal to the bond length, so that one polymer segment exactly fits into a lattice site. Solvent molecules are taken to have the same volume as a segment for simplicity.

At the upper boundary $z_{\mathrm{upp}}$, a reflecting boundary condition applies. This is implemented by forcing all density gradients to be zero at this boundary, i.e., $\varphi_{x}\left(z_{\text {upp }}+\right.$ $1)=\varphi_{x}\left(z_{\text {upp }}\right)$. Usually the value of $z_{\text {upp }}$ is taken very large, where the density gradients have vanished (bulk).

The density profile $\varphi(z)$ and related depletion thickness of PDMS polymer chains in cyclohexane next to the coated silica surface are modelled by SCMFT. The following assumptions are made to describe the PDMS polymer solution near the surface: i) the DMS segment size $0.25 \mathrm{~nm}$ is taken as the lattice length, ii) $N=1284 \mathrm{re}-$ peating units, iii) a flat wall represents the silica surface, iv) a cubic lattice is used, and iv) $\chi_{\mathrm{ps}}=0.4$ is applied [46] as the polymer-solvent interaction parameter.

Next, the interactions involving the silica wall are considered. First it is noticed that only the value of $\Delta \chi=$ $\chi_{\mathrm{ps}}-\chi_{\mathrm{pw}}$ and not the individual values of the solventwall $\chi_{\mathrm{sw}}(\equiv 0)$ and polymer-wall $\chi_{\mathrm{pw}}$ interaction parameters come into play. A larger $\Delta \chi$ implies that the polymers are more strongly attracted to the surface. In continuum models describing polymer densities at non-adsorbing surfaces $[9,56,57]$ and in GFVT, the condition $\varphi(z=0)=0$ is applied by default, see the appendix in [4] for a discussion.

The value for $\Delta \chi$ is implemented into theory for the phase behaviour as follows. From density profiles $\varphi(z)$, the adsorbed amount $\Gamma$ can be calculated from eq. (1) [2,3]. In case of a negative adsorption, a depletion thickness can be defined through $\delta=-\Gamma / \varphi_{b}$. Hence, it follows from eq. (1) that the depletion thickness can be determined from the density profiles according to $[2,3]$

$$
\delta=\int_{0}^{\infty}\left[1-\frac{\varphi(z)}{\varphi_{b}}\right] \mathrm{d} z .
$$

The computed result for $\delta$ at given $\Delta \chi$ is subsequently inserted into theory for the phase behaviour of mixtures of hard spheres and polymers, as explained below.

\subsubsection{Generalised free volume theory}

Free volume theory (FVT) for a colloid-polymer mixture is described by the semi-grand-canonical ensemble. The system of interest is held in osmotic equilibrium with a reservoir, that is separated from the system via a hypothetical membrane that allows permeation of equilibrium polymers and solvent molecules. The colloidal particles are however not allowed to enter the reservoir. The equilibrium polymer concentration in the system is set by the chemical potential of the polymer chains in the reservoir. The solvent can safely be considered as "background" [26, 31]. An expression is needed for the semi-grand potential $\Omega$ for the mixture of colloidal spheres and interacting polymers in order to be able to compute the thermodynamic properties of the mixture.

GFVT for the phase behaviour of a mixture of HS and excluded-volume polymers (EVP) $[31,40]$ takes into account the compression of the depletion layers in semidilute solutions and non-ideal contributions to the osmotic pressure, relevant near and above the polymer overlap concentration. In recent years, this approach has been refined to include the crossover from theta-solvent towards good-solvent conditions for the depletion interaction [58] as well as for the phase behaviour of colloid-polymer mixtures [59].

In GFVT, the semi-grand potential $\Omega$ is considered for a colloid-polymer mixture with volume $V$ in equilibrium with an external reservoir containing a solution of polymers only. The normalized semi-grand potential $\omega=\Omega v / k_{B} T V$ is introduced, with $v=4 \pi a^{3} / 3$ being the volume of a colloidal sphere. The fugacity of the polymer chains in the system is set by the polymer volume concentration $c^{R}$ in the reservoir. The polymer concentration $c$ in the system is related to $c^{R}$ through $c=\alpha c^{R}$, where $\alpha$ (see eq. (7)) is the free-volume fraction in the system. With $c^{*}$ denoting the polymer overlap concentration, the reduced polymer concentration is defined as $\phi_{p}=c / c^{*}=\varphi_{b} / \varphi_{b}^{*}$.

The normalized semi-grand potential

$$
\omega=f_{0}+\omega_{p}
$$

contains a HS and a polymer part. The HS part $f_{0}$ for a fluid is obtained from applying the Carnahan-Starling equation of state on the colloidal HS fluid [3] and the polymer contribution $\omega_{p}$ is given by

$$
\omega_{p}=-q^{-3} \int_{0}^{\phi_{p}} \alpha\left(\partial \widetilde{\Pi} / \partial \phi_{p}\right) \mathrm{d} \phi_{p}
$$


where the reduced osmotic pressure is given by $\widetilde{\Pi}=$ $\Pi v_{p} / k_{B} T$ with $v_{p}=4 \pi R_{g}^{3} / 3$.

The free-volume fraction $\alpha$ entering in eq. (6) is taken from the standard scaled-particle theory $[3,26]$ according to

$$
\alpha=(1-\phi) \mathrm{e}^{-\left[A \frac{\phi}{1-\phi}+B\left(\frac{\phi}{1-\phi}\right)^{2}+C\left(\frac{\phi}{1-\phi}\right)^{3}\right]},
$$

where $A=\left(1+\delta_{s} / a\right)^{3}-1, B=3 \delta_{s} / a^{2}\left(\delta_{s} / a+3 / 2\right)$, and $C=3 \delta_{s} / a^{3}$. Here, $\delta_{s}$ is the thickness of the depletion zone around the sphere with radius $a$.

For $\delta_{s}$ a result from Fleer et al. $[31,60]$ is used

$$
\delta_{s} / a=0.865 \frac{\delta}{\delta_{\text {ref }}}\left(q^{-2}+3.95 \phi_{p}^{1.54} q^{-2}\right)^{-0.44},
$$

in which curvature effects are included. The weak adhesion effect of the polymers is incorporated via the ratio $\delta / \delta_{\text {ref }}$ that is computed from eq. (4) for a dilute polymer solution at a wall, where $\delta_{\text {ref }}$ is the depletion thickness for the classical boundary condition $\varphi(z=0)=0$. It is implicitly assumed that the concentration and curvature dependence are not affected by the weak adhesion effect. The curvature effect is mainly geometrical; its effect is similar for instance when comparing ideal and interacting polymers [31]. With respect to the concentration dependence the effect will be tested in the Results and discussion section using exact numerical lattice computations. For the derivative of the osmotic pressure of a solution with EVP, the result [60]

$$
\partial \widetilde{\Pi} / \partial \phi_{p}=1+3.73 \phi_{p}^{1.31}
$$

is used.

Now all components to compute $\omega$ are available. Since the fugacity of the colloidal particles and osmotic pressure of the mixture can easily be obtained from $\omega$, it is straightforward to compute the binodal curves. Here, only colloidal gas-liquid coexistence is considered.

\subsection{Monte Carlo simulations}

Metropolis Monte Carlo simulations were performed in the canonical ensemble comprising fixed number of particles, volume and temperature. A cubic box with periodic boundary conditions in all three dimensions with varying box edges $L$ and a temperature of $298 \mathrm{~K}$ was used. Simulations were performed with 1000 spheres interacting via a HS radius of $37 \mathrm{~nm}$ at colloid and relative polymer volume fractions as indicated by table 1 . The interactions between the particles were defined by the depletion potential in which the depletion thickness was computed as described previously. The range of the depletion potential was set to $2.76 a$, corresponding to $q=0.38$. The weak adhesion effect is taken into account using the ratio $\delta / \delta_{\text {ref }}=0.71$. After equilibration, the simulations involved $10^{9}$ single particle trial moves and $10^{7}$ cluster trial moves.
Table 1. Overview of volume fraction of spherical particles $\phi$, examined lower and upper relative polymer concentrations $c / c^{*}$, and lower and upper estimate of relative polymer concentrations at onset of bulk instability obtained from Monte Carlo simulations.

\begin{tabular}{cccc}
\hline$L(\mathrm{~nm})$ & $\phi$ & $c / c^{*}$ (examined) & $c / c^{*}$ (instability) \\
\hline 3048 & 0.0075 & $1.5-2.1$ & $2.0-2.1$ \\
2419 & 0.015 & $1.5-2.0$ & $1.8-1.9$ \\
1920 & 0.030 & $1.5-2.0$ & $1.6-1.7$ \\
1524 & 0.060 & $1.4-2.0$ & $1.5-1.6$ \\
1210 & 0.090 & $1.3-2.0$ & $1.3-1.4$ \\
\hline
\end{tabular}

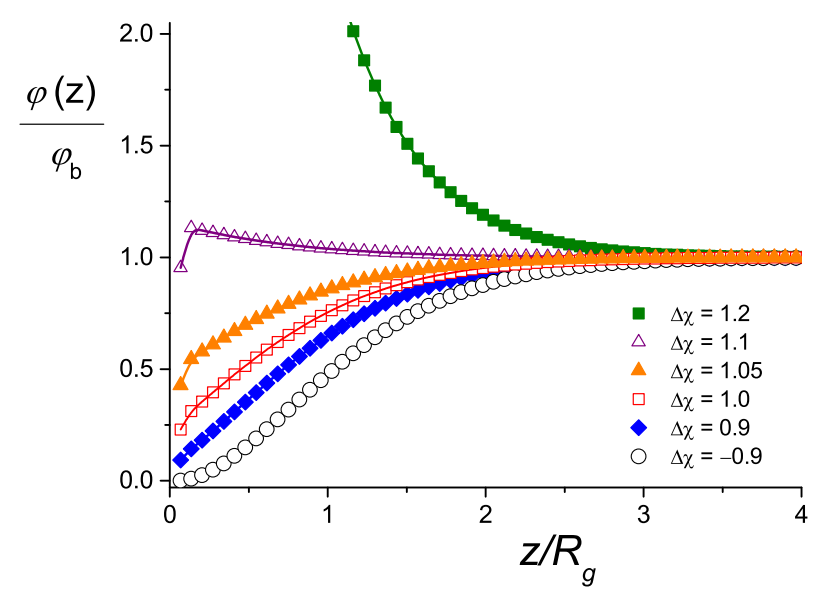

Fig. 3. Polymer density profiles $\varphi(z)$ normalized by the bulk density $\varphi_{b}$ as a function of the distance from the surface $z$ normalized by the polymer's radius of gyration for various values of $\Delta \chi$ with bulk density $\varphi_{b}=10^{-5}$ and polymer-solvent interaction parameter $\chi_{\mathrm{ps}}=0.4$. The density profiles are only weakly dependent on the values of $\varphi_{b}$ and $\chi_{\mathrm{ps}}$.

\section{Results and discussion}

In fig. 3 polymer density profiles normalized by the bulk density $\varphi_{b}$ are plotted as a function of $z$ for various values of $\Delta \chi$ as predicted by SCMFT. The bulk concentration was set at $\varphi_{b}=10^{-5}$. In particular, the condition $\varphi(z=0)=0$ used in classical depletion is achieved with $\Delta \chi \approx-0.9$ [4]. A more repulsive polymer-wall interaction ( $\Delta \chi$ goes down) hardly leads to a perceptible change of the density profile, whereas a less repulsive interaction ( $\Delta \chi$ goes up) weakens polymer depletion. At $\Delta \chi=0.9$ the density profile is still depletion-like, but the depletion zone is clearly reduced. Furthermore, a sharp transition from negative adsorption to positive adsorption appears near $\Delta \chi=1.1$, at which the adsorption energy balances the entropy loss of a polymer being close to the wall [2]. Finally, adsorption $(\Gamma>0)$ occurs at $\Delta \chi \gtrsim 1.2$.

The sensitivity of the density profiles on the value of $\Delta \chi$ displayed in fig. 3 demonstrates that it is essential to determine $\Delta \chi$ to quantify weak adhesion effects in case of some segment-surface adhesion. The value of $\Delta \chi$ has been deduced via atomic force microscopy measurements for a mixture of silica spheres mixed with PDMS in cy- 


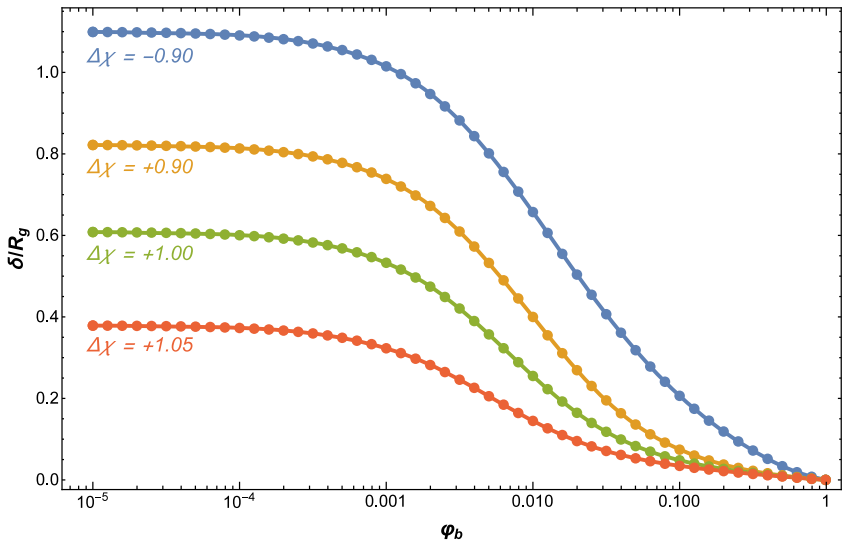

Fig. 4. Polymer concentration dependence of the depletion thickness from SCMFT for polymer chains with 1284 segments for a polymer-solvent interaction parameter $\chi_{\mathrm{ps}}=0.4$. Results for various values of $\Delta \chi$ are plotted.

clohexane by Wijting et al. [46]. They studied the interaction between a silica sphere coated with stearyl alcohol and a flat silica surface in cyclohexane as a function of distance between the surfaces and PDMS concentration in detail. A weaker depletion force was measured than predicted based on classical depletion theory for the interaction based on the Asakura-Oosawa-Vrij interaction potential $[7,11]$, which could be explained by a weak adhesion of polymer chains, employing a value of $\Delta \chi \approx 0.9$ [46].

In fig. 4 the depletion thickness (normalised by the radius of gyration $R_{g}$ of the polymer chains) is plotted as a function of the bulk polymer concentration for various values of $\Delta \chi$. Again, it is clear that overall the depletion thickness drops as $\Delta \chi$ increases above a value of -0.9 . When increasing the bulk concentration the depletion thickness starts to decrease near the polymer overlap concentration due to a high osmotic pressure pushing the polymer chains closer to the wall [4]. This polymer concentration dependence is similar and therefore eq. (8) is expected to still be a good approximation for interacting polymers that are weakly adhesive.

Next, theoretical predictions and Monte Carlo simulations are now confronted with experimental phase diagrams of colloid-polymer mixture containing sterically stabilized silica spheres mixed with PDMS polymers in cyclohexane.

The phase diagram for a mixture of silica spheres and PDMS polymers in cyclohexane obtained in various manners is drawn in fig. 5. First, the binodal colloidal gasliquid coexistence curve has been measured experimentally (filled symbols) [49]. Second, three theoretical predictions are presented: i) FVT comprising HS representing the colloid and penetrable hard spheres (PHS) representing the polymer coil (dotted curve), ii) GFVT with an improved description of the polymer coil (dashed curve) and iii) depletion theory with weak adhesion accounted for based on GFVT with the depletion thickness calculated from SCMFT (solid curve). The prediction of the two first theoretical approaches involving classical depletion do not differ much and underestimate the polymer concentration

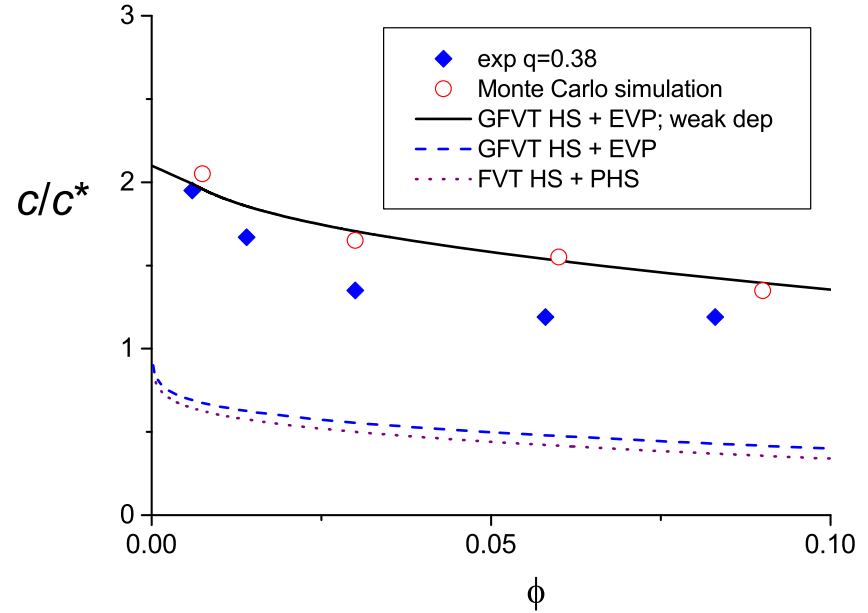

Fig. 5. Phase diagram of cyclohexane solution containing silica spheres coated with stearyl alcohol with radius $a=37 \mathrm{~nm}$ and PDMS polymers with a weight-averaged molar mass of 95 $\mathrm{kg} / \mathrm{mol}$ and a radius of gyration $R_{g}=14.4 \mathrm{~nm} . q=R_{g} / a=$ 0.38 . Note that the free volume theory predictions are binodals, while the MC simulation results are spinodals.

at the binodal by more than a factor 2 . In contrast, the weak adhesion prediction is very close to the experimental binodal curve. Finally, results of Monte Carlo simulations employing a depletion potential with weak adhesion incorporated, also given in fig. 5 (open circles), basically verified the theoretical prediction of the depletion theory with weak adhesion based on GFVT. Table 1 provides narrow ranges where solution instabilities appears. Snapshots of equilibrated configurations from the Monte Carlo simulations are shown in fig. 6 for $\phi=0.06$ at $c / c^{*}=1.4$ and $c / c^{*}=2.0$. These snapshots illustrate the transition in the change of the configurations of the spheres. For $c / c^{*}=1.4$ the particles tend to be close to one another, but are still distributed over the entire phase space. Clear clustering effects can be observed for $c / c^{*}=2.0$. As an illustration representative results for the structure factor $S(Q)$ are plotted in fig. 7. It follows that the structure factor at small wave vectors $(Q)$ strongly increases with relative polymer concentration. At the spinodal, $S(Q=0)$ formally diverges, but for a finite system of $N$ particles, $S(Q)$ cannot exceed $N$ for a finite system in the limit $Q \rightarrow 0$. In the simulations it is of course not possible to find $Q \rightarrow 0$ because the box size is finite. Therefore we focused on $S(Q=2 \pi / L)$, with $L$ the box size. Hence the spinodal points were determined by examining the polymer concentration at which the structure factor approaches $S(Q)=N$ at $Q=2 \pi / L$. At such a condition only a single large aggregate is formed, as shown in the snapshots. It is hence noted that the spinodals are approximate. Moreover, the simulations are based on assuming pairwise additivity of the effective interactions, while free volume theory explicitly accounts for inclusion of the polymers as depletant. In fig. 5 binodal and spinodal curves are mixed; nevertheless the main conclusions are expected to hold. 

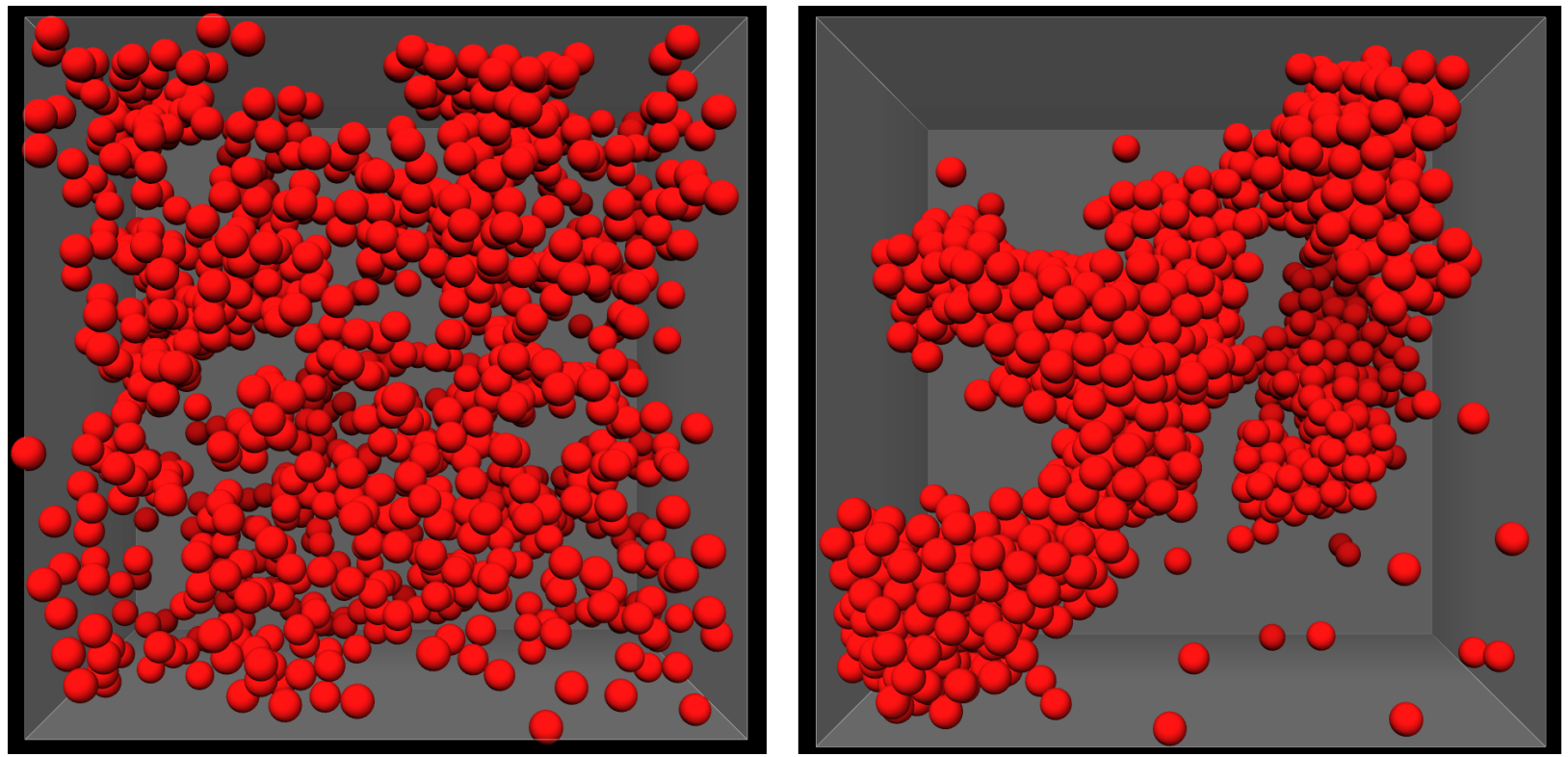

Fig. 6. Snapshots of equilibrated Monte Carlo simulation configurations for $\phi=0.060$ below (a) and above (b) phase instability.

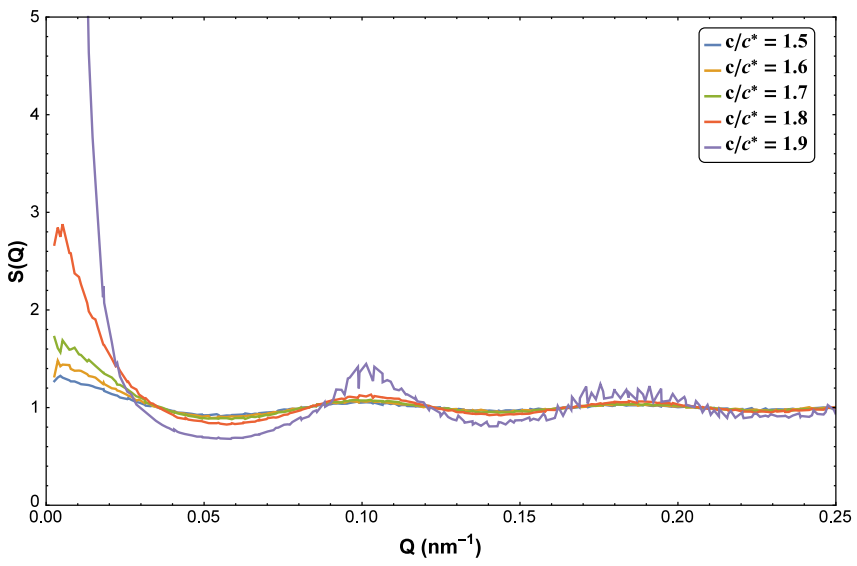

Fig. 7. Structure factor $S(Q)$ as a function of wave vector $Q$ at $\phi=0.015$ for relative polymer concentrations as indicated.

The theoretical predictions of our depletion theory with weak adhesion incorporated were further compared to experimental data by Bodnár and Oosterbaan [43] for a chemically similar system composed of silica spheres and PDMS polymers in cyclohexane. Their study comprised silica particles and polymers of different sizes giving a size ratio $q=0.49$. Figure 8 shows their experimental phase diagram up to colloid volume fraction $\phi \approx 0.5$ and our theoretical predictions. Again, FVT and GFVT with classical depletion severely underestimates the measured coexistence curve, while the prediction by the depletion theory with weak adhesion incorporated is much closer to the experimental results.

It follows that inclusion of the weak adhesion effect into depletion theory allows to provide reasonably accurate predictions of the phase coexistence curves for systems where weak adhesion occurs. A more accurate description

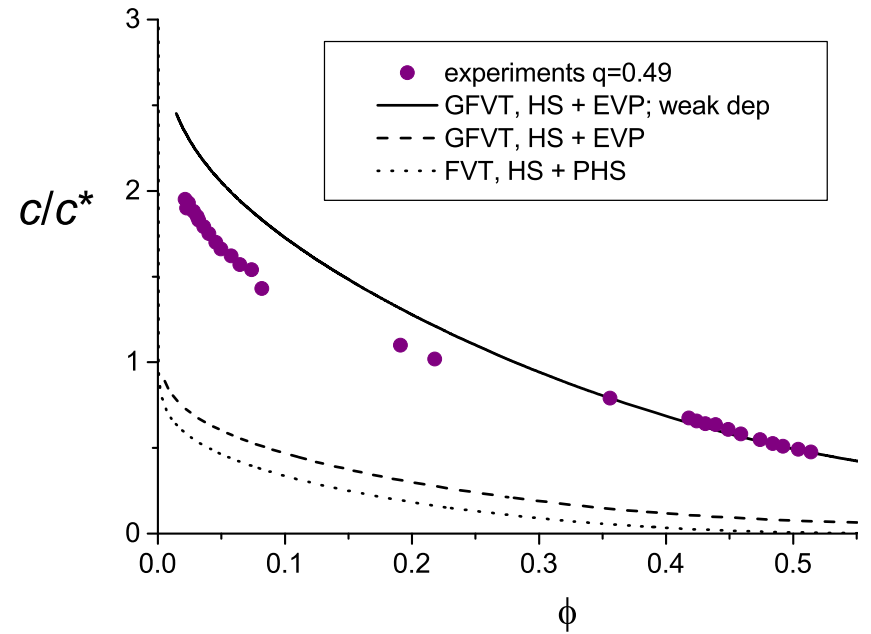

Fig. 8. Phase diagram of cyclohexane solution with silica spheres with radius $a=47 \mathrm{~nm}$ and PDMS polymers with a weight-averaged molar mass of $204 \mathrm{~kg} / \mathrm{mol}$ and a radius of gyration $R_{g}=23 \mathrm{~nm} . q=R_{g} / a=0.49$. Experimental data from ref. [43].

of the polymeric excluded-volume effect $[58,59]$ plus accounting for size dispersity $[45,61]$ will probably provide even more precise predictions of the phase diagram. Probably, colloid size dispersity effects can explain the small but significant deviations between theory and experiment that can still be observed in figs. 5 and 8 . Fasolo and Sollich [45] showed that more dispersity in colloid size leads to an upwards shift of colloidal gas-liquid coexistence curves and that colloid size dispersity disfavours (colloidal) fluidsolid versus gas-liquid phase separation. In another paper Fasolo and Sollich [61] demonstrated that polymer size dispersity leads to nearly size dispersity-independent phase 
diagrams, provided the comparison is done at using the same mass-averaged polymer molar mass.

It is noted that PRISM theory [34] also allows to quantify the details of depletion effects with weak adhesion incorporated on the particle and structure level. To compute full phase diagrams using PRISM is rather complicated, while free volume theory is rather straightforward. It becomes clear that it is essential to be able to determine the thermodynamic interactions [62] between the different compounds (surface, solvent, polymer segment) to be able to account for adhesion effects in detail when it is required to go beyond pure hard-core interactions between colloidal particles and polymer segments.

\section{Conclusions}

In this paper, a depletion theory incorporating weak adhesion is presented for the phase behaviour of a dispersion of hard-sphere colloids and non-adsorbing polymer with excluded-volume interactions for arbitrary size ratio $q=R / a$. Semi-quantitative agreement between the new theory and experimental data is found for two different systems comprising silica spheres and PDMS in cyclohexane, whereas classical depletion theory provides binodal curves with polymer concentrations off by more than a factor of two. It follows that care should be taken when interpreting depletion effects in colloid-polymer mixtures since the interaction potential, the resulting phase behaviour and the dynamics of the mixture depend on the details of the polymer-colloid surface interactions. Hence one should aim at carefully analysing whether there is positive or negative adsorption. When there is negative adsorption care should be taken that the strength of adhesion of the polymers at the surface is quantified.

\section{Author contribution statement}

Remco Tuinier and Samia Ouhajji developed the theoretical approach. Remco Tuinier computed the self-consistent mean-field results and free volume theory results and wrote the manuscript. Samia Ouhajji performed the experiments to determine the experimental phase diagram in fig. 5. Per Linse and Samia Ouhajji made the Monte Carlo simulations. Per Linse contributed to useful discussions on the theory and initiated the computer simulations. Per Linse and Samia Ouhajji co-wrote the manuscript.

We thank Albert Philipse, Frans Leermakers, Tommy Nylander and Lennart Piculell for useful discussions.

Open Access This is an open access article distributed under the terms of the Creative Commons Attribution License (http://creativecommons.org/licenses/by/4.0), which permits unrestricted use, distribution, and reproduction in any medium, provided the original work is properly cited.

\section{References}

1. W.B. Russel, D.A. Saville, W.R. Schowalter, Colloidal Dispersions (Cambridge Universtity Press, USA, 1999).

2. G.J. Fleer, M.A. Cohen Stuart, J.M.H.M. Scheutjens, T. Cosgrove, B. Vincent, Polymers at Interfaces (Chapman and Hall, New York, 1993).

3. H.N.W. Lekkerkerker, R. Tuinier, Colloids and the Depletion Interaction (Springer, 2011).

4. G.J. Fleer, A.M. Skvortsov, R. Tuinier, Macromolecules 36, 7857 (2003).

5. M. Surve, V. Pryamitsyn, V. Ganesan, Langmuir 22, 969 (2006).

6. J.B. Hooper, K.S. Schweizer, Macromolecules 39, 5133 (2006).

7. S. Asakura, F. Oosawa, J. Chem. Phys. 22, 1255 (1954).

8. W.C.K. Poon, J. Phys.: Condens. Matter 14, R859 (2002).

9. E. Eisenriegler, J. Chem. Phys. 79, 1052 (1983).

10. S. Asakura, F. Oosawa, J. Polym. Sci. 33, 183 (1958).

11. A. Vrij, Pure Appl. Chem. 48, 471 (1976).

12. D. Kleshchanok, R. Tuinier, P.R. Lang, J. Phys.: Condens. Matter 20, 073101 (2008).

13. R. Tuinier, T.-H. Fan, T. Taniguchi, Curr. Opin. Colloid Interface Sci. 20, 66 (2015).

14. J.-L. Doublier, C. Garnier, D. Renard, C. Sanchez, Curr. Opin. Colloid Interface Sci. 5, 202 (2000).

15. B.R. Saunders, M.L. Turner, Adv. Colloid Interface Sci. 138, 1 (2008)

16. P.R. Sperry, H.B. Hopfenberg, N.L. Thomas, J. Colloid Interface Sci. 82, 62 (1981).

17. F. Leal-Calderon, J. Bibette, J. Biais, Europhys. Lett. 23, 653 (1993).

18. S.M. Ilett, A. Orrock, W.C.K. Poon, P.N. Pusey, Phys. Rev. E 51, 1344 (1995).

19. N.A.M. Verhaegh, J.S. van Duijneveldt, J.K.G. Dhont, H.N.W. Lekkerkerker, Physica A 230, 409 (1996).

20. M.A. Faers, P.F. Luckham, Langmuir 13, 2922 (1997).

21. E.H.A. de Hoog, H.N.W. Lekkerkerker, J. Phys. Chem. B 103, 5274 (1999)

22. Z. Zhang, J.S. van Duijneveldt, Langmuir 22, 63 (2006).

23. A. Moussaïd, W.C.K. Poon, P.N. Pusey, M.F. Soliva, Phys. Rev. Lett. 82, 225 (1999).

24. R. Tuinier, P.A. Smith, W.C.K. Poon, S.U. Egelhaaf, D.G.A.L. Aarts, H.N.W. Lekkerkerker, G.J. Fleer, Europhys. Lett. 82, 68002 (2008).

25. J. Sabin, A.E. Bailey, G. Espinosa, B.J. Frisken, Phys. Rev. Lett. 109, 195701 (2012).

26. H.N.W. Lekkerkerker, W.C.K. Poon, P.N. Pusey, A. Stroobants, P.B. Warren, Europhys. Lett. 20, 559 (1992).

27. M. Schmidt, H. Löwen, J.M. Brader, R. Evans, Phys. Rev. Lett. 85, 1934 (2000).

28. E.J. Meijer, D. Frenkel, J. Chem. Phys. 100, 6873 (1994).

29. A. Moncho-Jordá, A.A. Louis, P.G. Bolhuis, R. Roth, J. Phys.: Condens. Matter 15, S3429 (2003).

30. M. Dijkstra, R. van Roij, R. Roth, A. Fortini, Phys. Rev. E 73, 041404 (2006).

31. G.J. Fleer, R. Tuinier, Adv. Colloid Interface Sci. 143, 1 (2008).

32. M. Surve, V. Pryamitsyn, V. Ganesan, J. Chem. Phys. 122, 154901 (2005).

33. V. Ganesan, C.J. Ellison, V. Pryamitsyn, Soft Matter 6, 4010 (2010). 
34. M. Fuchs, K.S. Schweizer, J. Phys.: Condens. Matter 14, R239 (2002).

35. S. Ramakrishnan, M. Fuchs, K.S. Schweizer, C.F. Zukoski, J. Chem. Phys. 116, 2201 (2002).

36. S. Ramakrishnan, M. Fuchs, K.S. Schweizer, C.F. Zukoski, Langmuir 18, 1082 (2002).

37. A.A. Louis, P.G. Bolhuis, E.J. Meijer, J.P. Hansen, J. Chem. Phys. 116, 10547 (2002).

38. A.A. Louis, P.G. Bolhuis, E.J. Meijer, J.P. Hansen, J. Chem. Phys. 117, 1893 (2002).

39. P.G. Bolhuis, A.A. Louis, J.P. Hansen, Phys. Rev. Lett. 89, $128302(2002)$

40. D.G.A.L. Aarts, R. Tuinier, H.N.W. Lekkerkerker, J. Phys.: Condens. Matter 14, 7551 (2002).

41. G. D'Adamo, A. Pelissetto, C. Pierleoni, Macromolecules 49, 5266 (2016).

42. P.G. Bolhuis, D.A. Kofke, Phys. Rev. E 54, 634 (1996).

43. I. Bodnár, W.D. Oosterbaan, J. Chem. Phys. 106, 7777 (1997).

44. J.S. Van Duijneveldt, K. Mutch, J. Eastoe, Soft Matter 3, 155 (2007).

45. M. Fasolo, P. Sollich, J. Chem. Phys. 122, 074904 (2005).

46. W.K. Wijting, W. Knoben, N.A.M. Besseling, F.A.M. Leermakers, M.A. Cohen Stuart, Phys. Chem. Chem. Phys. 6, 4432 (2004).

47. A.A. Louis, H. Allahyarov, H. Löwen, R. Roth, J. Chem. Phys. 65, 061407 (2002).

48. A.K. Van Helden, J.W. Jansen, A. Vrij, J. Colloid Interface Sci. 81, 354 (1981).
49. S. Ouhajji, T. Nylander, L. Piculel, R. Tuinier, P. Linse, A.P. Philipse, Soft Matter 12, 3963 (2016).

50. B. Vincent, Colloids Surf. 50, 241 (1990).

51. N. Kuwahara, T. Okazawa, M. Kaneko, J. Polym. Sci., Part C, Polym. Symp. 23, 543 (1968).

52. J.M.H.M. Scheutjens, G.J. Fleer, J. Phys. Chem. 83, 1619 (1979).

53. C.M. Wijmans, E.B. Zhulina, G.J. Fleer, Macromolecules 27, 3238 (1994).

54. G.H. Fredrickson, V. Ganesan, F. Drolet, Macromolecules 35, 16 (2002).

55. F.A.M. Leermakers, J.C. Eriksson, J. Lyklema, in Fundamentals in Colloid and Interface Science, Vol. 5 (Elsevier, Amsterdam, 2005).

56. E. Eisenriegler, A. Hanke, S. Dietrich, Phys. Rev. E 54, 1134 (1996).

57. E. Eisenriegler, Phys. Rev. E 55, 3116 (1997).

58. G. D'Adamo, A. Pelissetto, C. Pierleoni, Mol. Phys. 111, 3372 (2013).

59. G. D'Adamo, A. Pelissetto, C. Pierleoni, J. Chem. Phys. 141, 024902 (2014).

60. G.J. Fleer, A.M. Skvortsov, R. Tuinier, Macromol. Theory Simul. 16, 531 (2007).

61. M. Fasolo, P. Sollich, J. Phys.: Condens. Matter 17, 797 (2005).

62. J.J. Gutiérrez-Sevillano, K. Leonhard, J.P.J.M. van der Eerden, T.J.H. Vlugt, G.J.P. Krooshof, Ind. Eng. Chem. Res. 54, 2214 (2015). 